\title{
Extreme-Value Statistics for Testing Dark Energy
}

\section{Simone Aiola ${ }^{1}$, Arthur Kosowsky and Bingjie Wang}

Department of Physics and Astronomy \& Pitt-PACC, University of Pittsburgh, PA 15260

${ }^{1}$ email: sia21@pitt.edu

\begin{abstract}
The integrated Sachs-Wolfe effect was recently detected at a level of $4.4 \sigma$ by Granett et al. (2008), by stacking compensated CMB temperature patches corresponding to superstructures in the universe. We test the reported signal using realistic gaussian random realizations of the CMB sky, based on the temperature power spectrum predicted by the concordance $\Lambda$ CDM model. Such simulations provide a complementary approach to the largely used N-body simulations and allow to include the contaminant effects due to small-scale temperature fluctuations. We also apply our pipeline to foreground-cleaned CMB sky maps using the Granett et al. (2008) voids/clusters catalog. We confirm the detection of a signal, which depart from the null hypothesis by $3.5 \sigma$, and we report a tension with our theoretical estimates at a significance of about $2.5 \sigma$.
\end{abstract}

Keywords. cosmic microwave background, large-scale structure of universe, methods: numerical

\section{Introduction}

The current state of accelerated expansion of the universe, driven by Dark Energy (DE), leaves an imprint in the large-scale cosmic structure (at redshifts $z \lesssim 2$ ), as well as on the CMB temperature fluctuations. The time-evolving gravitational potential weakly redshifts/blueshifts microwave photons, traveling from the last scattering surface to us. This effect is known as late-time integrated Sachs-Wolfe effect (late-ISW, hereafter) (Sachs \& Wolfe (1967)), and its detection represents an independent test for DE, and in principle a useful probe to characterize its properties and dynamics.

In 2008, Granett et al. (2008) (GNS08, hereafter) reported the strongest detection of the late-ISW, at a significance level of $4.4 \sigma$. This strong detection exploited a novel technique involving analysis of stacked CMB patches centered on 100 superstructures, which are detected in SDSS DR6 in a redshift range $0.4<z<0.75$. The photometric signal averaged over a $4^{\circ}$ compensated disk corresponds to $\left\langle T_{m}^{G N S 08}\right\rangle=9.6 \pm 2.2 \mu \mathrm{K}$. This was confirmed by the Planck collaboration (PLK13, hereafter), which reports a value of $\left\langle T_{m}^{P L K 13}\right\rangle=8.7 \pm 2.2 \mu K$ (Planck Collaboration, XIX, 2013).

It has been argued by Flender et al. (2013) (FHN13, hereafter), that this strong signal is in tension with the underlying $\Lambda \mathrm{CDM}$ model. Analytical estimates of the stacked lateISW signal (only), in a comoving volume that corresponds to what probed by GNS08 (ISW-SDSS, hereafter), predict an average signal of $\left\langle T_{m}^{F N^{13}}\right\rangle=2.27 \pm 0.14 \mu \mathrm{K}$. This estimate is in tension with the GNS08 results at a level bigger than $3.0 \sigma$. This discrepancy is confirmed in the same work using computationally intense N-body simulations, which may have problems related to the size of the simulations boxes.

The GNS08 technique relies on extreme-value statistics, which may be easily reveal small departures from the $\Lambda$ CDM model. On the other hand, an efficient control of the systematics and careful consistency checks between data and simulations are required. In this work, we aim to test this prerequisites, which are fundamental to promote the 

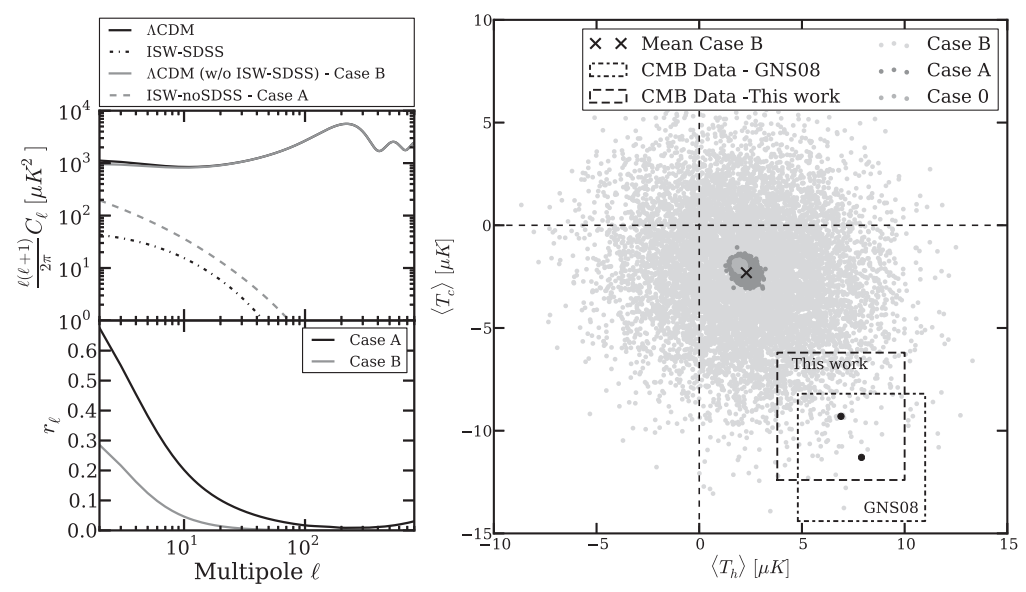

Figure 1. (Left panel) CMB temperature auto-spectra, assuming concordance $\Lambda$ CDM model. The late-ISW is broken up into two different components, in order to show the relative amplitudes compared to the fluctuations originated at $z \sim 1100$. The correlation coefficients $r_{\ell}=C_{\ell}^{X Y} / \sqrt{C_{\ell}^{X X} C_{\ell}^{Y Y}}$ are plotted in the bottom part, showing the importance of the crosscorrelation between the probed ISW-SDSS and the other contributions to the CMB map. (Right panel) summary of the results from this analysis. From the simulations, we notice that the effect of the correlations slightly shifts the expectation values. However, the effect of the small-scale fluctuations and the cosmic variance drastically change the intrinsic signal-to-noise ratio of such a measurement. The results from the analysis of the CMB map with our pipeline are compared with the previous analysis of GNS08.

GNS08 measurement from a simple detection to a cosmological probe of the late-time universe.

\section{A Simple Description of the CMB Sky}

The large-scale CMB sky is well described by a gaussian random field, which statistical properties are fully specified by the usual power spectrum $C_{\ell}^{T T}$. Specifically, different physical processes cause temperature fluctuations at different angular scales. Consequently, the CMB sky map can be considered as the sum of different correlated gaussian fields, each separately described by auto-spectra, and the cross-spectra define the correlations between them. In the left panel of Fig. 1, we show the auto-spectra of the ISW-SDSS (corresponding to the signal we aim to detect) and the auto-spectra of the late-ISW coming from neighboring regions of what it is probed by GNS08, as well as the temperature fluctuations generated at the last scattering surface. We also plot (in the bottom part of the same panel) the normalized correlation coefficients between two different components. For a 2 -component sky, we need only two coefficients $A_{\ell}$ and $B_{\ell}$ :

$$
A_{\ell}=\left|B_{\ell}\right|\left|F_{\ell}\right| \sqrt{C_{\ell}^{1,1}}\left(1+\frac{C_{\ell}^{1,2}}{C_{\ell}^{1,1}}\right), \quad B_{\ell}=\left|B_{\ell}\right|\left|F_{\ell}\right|\left(C_{\ell}^{2,2}-\frac{\left(C_{\ell}^{1,2}\right)^{2}}{C_{\ell}^{1,1}}\right)^{1 / 2}
$$

where $F_{\ell}$ is the Legendre transform of the $4^{\circ}$ compensated TopHat filter used in the GNS08 analysis. From such coefficients, we can generate two temperature maps and simply co-add them. This algorithm gives a realistic realization of a filtered CMB sky, which is properly correlated with a given realization of the ISW-SDSS map (separately used in the analysis). 


\section{Numerical Simulations and CMB Data Analysis}

From the assumption that the late-ISW is traced by superstructures in the universe, we can use the ISW-SDSS map (previously generated) as a tracer of clusters/voids (hot/cold spots) catalog. Specifically, we consider an ensemble of 5000 simulated skies, focusing only on $20 \%$ of the total sky area (GNS08 sky fraction). The pipeline we adopted can be described as follows:

(a) we identify and rank cold and hot spots in a randomly-generated filtered ISWSDSS map (called tracer map);

(b) we generate filtered CMB temperature map, which contains different temperature components. These components are properly correlated with the previous tracer map (called temperature map);

(c) we average the pixels temperatures of the temperature map, which correspond to the 50 top-ranked hot spots, $\left\langle T_{h}\right\rangle$, and 50 top-ranked cold spots, $\left\langle T_{c}\right\rangle$, (identified in the tracer map), separately;

(d) we also compute the mean temperature as $\left\langle T_{m}\right\rangle=\left(\left\langle T_{h}\right\rangle-\left\langle T_{c}\right\rangle\right) / 2$;

We consider different cases, which differ from each other depending on the components we consider in the temperature map: (Case 0) the temperature map has only the ISWSDSS component (same as tracer map). (Case A) the temperature map has only lateISW, but integrated over the whole redshift range $0<z<10$. (Case B) the temperature map actually describes the CMB sky (comparable with GNS08, PLK13 and our analysis on $\mathrm{CMB}$ data). The results of our simulations are shown in the right panel of Fig. 1. For the interesting case (Case B), which best describes the observed sky, we find $\left\langle T_{h}\right\rangle=$ $-\left\langle T_{c}\right\rangle=2.30 \pm 3.1 \mu \mathrm{K}$ and $\left\langle T_{m}\right\rangle=2.32 \pm 2.32 \mu \mathrm{K}$. We notice that this realistic case is in agreement with previous estimates by FHN13. However, we can argue that the filter (which suppresses the largest temperature fluctuations), removes possible issues related to the modes larger than the size of the N-body simulations. A different (and possibly more suitable) filter may not satisfy such a condition. In order to test the simulative pipeline against the data, we also reanalyze the CMB data using our harmonic-space filtering procedure and the GNS08 catalog. We find great consistency with the previous works by GNS08 and PLK13. Specifically, we report the following temperature values $\left\langle T_{h}\right\rangle=6.89 \pm 3.1 \mu K,\left\langle T_{c}\right\rangle=-9.33 \pm 3.1 \mu K$ and $\left\langle T_{m}\right\rangle=8.11 \pm 2.32 \mu K$.

\section{Conclusions and Future Prospectives}

In this work, based on the upcoming paper (Aiola et al. 2014), we presented a detailed analysis of the stacked late-ISW signature, within flat $\Lambda$ CDM cosmology. From the analysis of available CMB sky maps, we report a departure from the null signal at a level of $3.5 \sigma$ level, which shows a $2.5 \sigma$ tension with the expected maximum late-ISW signal. For the future, we plan to optimize and use this method as a cosmological probe for the equation of state of Dark Energy. Methods based on extreme-value statistics are intrinsically sensitive to different expansion histories, therefore useful to explore the parameter space.

\section{References}

Aiola, S., Kosowsky, A., \& Wang, B. 2014, in preparation

Flender, S., Hotchkiss, S., \& Nadathur, S. 2013, JCAP, 2, 013, 13

Granett, B. R., Neyrinck, M. C., \& Szapudi, I. 2008, ApJ (Letters), 683, L99

Planck Collaboration 2013, arXiv:1303.5079

Sachs, R. K. \& Wolfe, A. M. 1967, ApJ, 147, 73 